\author{
Department of Economics \\ and \\ Institute for Policy Analysis \\ University of Toronto \\ 150 St. George St. \\ Toronto ON M5S 3G7 Canada \\ WORKING PAPER \\ NUMBER UT-ECIPA-msmart-96-01
}

March 12, 1996

\title{
Competitive insurance markets with two unobservables ${ }^{1}$
}

\author{
Michael Smart \\ University of Toronto \\ and \\ Stanford University
}

(c) 1996 by Michael Smart
Department of Economics
University of Toronto

ISSN 0829-4909

Author's e-mail: msmart@epas.utoronto.ca

On-line version: http://www.epas.utoronto.ca:8080/ecipa/wpa.html 


\begin{abstract}
We study a screening game in a competitive insurance market, in which insurance customers differ with respect to both accident probability and degree of risk aversion. It is shown that indifference curves of customers in different risk classes cross exactly twice: thus the single crossing property does not hold. The existence of a unique reactive equilibrium is demonstrated. This equilibrium may be markedly different from the Paretodominant separating equilibrium that exists when single crossing holds. In particular, types may be pooled in equilibrium, so that cross-subsidization occurs. Moreover, insurance firms can earn positive expected profit in equilibrium, despite the usual assumption of Bertrand-like price-competition among firms. We study the implications of the model for the efficiency of market equilibrium and for the effects of rate-of-return regulation of insurance firms.
\end{abstract}




\section{Introduction}

How do competitive insurance markets function when insurers cannot observe ex ante the loss probabilities of potential customers? In canonical theoretical models, ${ }^{2}$ departures from full insurance (such as deductibles) are used to induce customers to reveal their private information. While the precise nature of market outcomes depends on the equilibrium concept employed, risk classes are typically fully separated in equilibrium, with high-risk and low-risk customers choosing different contracts. In these models, insurance firms earn zero expected profit in competitive equilibrium, and the equilibrium allocation satisfies a notion of information-constrained efficiency.

When potential customers are identical in every respect other than accident probability, then the single crossing property obtains - low risk customers are more willing to accept less insurance (a larger deductible) than high-risk customers - and the traditional results follow naturally. But when customers differ in other ways as well, the single crossing condition may not hold, and the role of incomplete insurance in separating risk classes becomes less apparent.

This paper considers a model of competitive screening in insurance markets in which insurance customers differ in more than one characteristic, and it investigates the implications of the model for the existence and properties of equilibrium. Customers differ with respect to both accident probabilities and degree of risk aversion, each of which is unobservable to insurance firms. ${ }^{3}$ It is shown that indifference curves of customers with low accident probability but high risk aversion cross twice the indifference curves of customers with high accident probability but low risk aversion (if they cross at all). Consequently, preferences are "double crossing," rather than single crossing.

It is shown that a unique reactive equilibrium exists for all specifications of preferences satisfying weak conditions. ${ }^{4}$ Thus, the existence and uniqueness results that are standard under one-dimensional single crossing carry over to the two-dimensional case considered here. The equilibrium may, however, be significantly different.

The nature of the equilibrium allocation depends on the precise specification of preferences for insurance customers. For some preferences, different risk classes may be pooled at a single contract in equilibrium; thus cross-subsidization may occur. Specifically, some low risk individuals pay more and some high risk individuals pay less than the actuarial value of their insurance policies. For some preferences, moreover, firms may earn strictly positive profits on some of the contracts offered in equilibrium. In contrast, when single

\footnotetext{
${ }^{1}$ Thanks to Douglas Bernheim, Peter Hammond, Kenneth Judd and Joseph Stiglitz for their advice. (The usual disclaimer applies.) This research was supported in part by a Sloan Foundation Dissertation Fellowship.

${ }^{2}$ Many of these models, which include Rothschild and Stiglitz (1976); Wilson (1977); Miyazaki (1977); Riley (1979); Myerson (1983), and Cho and Kreps (1987), inter alia, are developed in other contexts but apply naturally to the case of insurance markets.

${ }^{3}$ Thus agents' types are two-dimensional, whereas their signals (deductibles) are one-dimensional. Quinzii and Rochet (1985) consider the case in which types and signals are both $n$-dimensional. As they observe, separating equilibria are far more likely to exist in that case than when signals are of lower dimension than types.

${ }^{4}$ Reactive equilibrium, introduced by Riley (1979), is defined below. The qualitative results derived for reactive equilibrium also obtain under other solution concepts, including the Cho-Kreps Intuitive criterion applied to the corresponding signalling game.
} 
crossing holds, all contracts earn zero profits in equilibrium.

Positive equilibrium profit occurs in the model because, when preferences are not single crossing, it may be infeasible for insurers to reduce premiums without violating incentive constraints and attracting high-risk customers to contracts intended for low-risk customers. Thus the model provides an alternative explanation for the observed recent failure of some firms to exit from liability insurance lines in which premiums have been capped or rolled back by state regulators. Researchers have typically regarded this phenomenon as inconsistent with competitive equilibrium, and have adduced various political-economic explanations for firms' behaviour. ${ }^{5}$ But this paper suggests that positive profit may well be consistent with competitive equilibrium, if preferences are not single crossing, and so the result that firms do not exit markets in which premiums are suppressed may not be so anomalous.

\section{Insurance markets and information}

\subsection{Model structure}

Consider an individual who faces a risk of a loss in money income. The stochastic structure is simple. There are two states of the world; in the "good" state, which occurs with (exogenous) probability $\pi$, no loss occurs, and the individual's income before insurance is $x>0$. In the "bad" state, which occurs with probability $1-\pi$, the individual experiences a loss of $b$ and income is $x-b \geq 0$. The monetary parameters $x$ and $b$ are publicly known; the accident probabilities are, obversely, private information. In addition, the agent is risk averse. As with accident probability, an individual's degree of risk aversion cannot be verifiably observed by insurance companies $e x$ ante.

Insurance firms offer individuals contracts which yield (insured) income of $y$ if no accident occurs and $y-c$ if an accident occurs. Equivalently, customers pay firms a "premium" $x-y \geq 0$ ex ante, and, if an accident occurs, the firm compensates the individual for the loss, minus the "deductible" amount $c \leq b$.

The individual may be of one of two possible types $i=L, H$. (The relevance of the individual's type will be explained shortly.) The individual is a Neumann-Morgenstern expected utility maximizer, with utility function

$$
U^{i}(c, y)=\pi_{i} u\left(y, \theta_{i}\right)+\left(1-\pi_{i}\right) u\left(y-c, \theta_{i}\right),
$$

where $u$ is strictly concave and twice-continuously differentiable in its first argument and $\theta_{i}$ is a scalar indexing the degree of absolute risk aversion $a$. Specifically,

$$
a^{i}(y)=-\frac{u_{11}\left(y, \theta_{i}\right)}{u_{1}\left(y, \theta_{i}\right)}, \quad i=L, H
$$

and it is assumed that $a^{H}(y) \geq a^{L}(y)$ for all $y$.

\footnotetext{
${ }^{5}$ A typical explanation is that firms face fixed costs of entering an insurance line, and expect future regulatory changes that will restore profitability. Consequently, exit from a regulated line may not maximize long-run profit. See Harrington (1992), for example.
} 
The individual's marginal rate of substitution between the deductible and income is denoted

$$
\left.\sigma^{i}(c, y) \equiv \frac{d c}{d y}\right|_{\bar{U}^{i}}=1+\frac{\pi_{i}}{1-\pi_{i}} \mu^{i}(c, y)
$$

where

$$
\mu^{i}(c, y)=\frac{u_{1}\left(y, \theta_{i}\right)}{u_{1}\left(y-c, \theta_{i}\right)}, \quad i=L, H
$$

is the individual's marginal rate of substitution between income in two states given a contract $(c, y)$.

The pair $i \equiv(\pi, \theta)$ is referred to as the individual's type. For simplicity, it is assumed that the informed agent may have one of two types, so that $i \in T \equiv\left\{\left(\pi_{L}, \theta_{L}\right),\left(\pi_{H}, \theta_{H}\right)\right\}$, with $0<\pi_{L}<\pi_{H}<1$ and $0<\theta_{L}<\theta_{H}$. Thus insurance customers are either highprofit with high risk aversion or are low-profit with low risk aversion. ${ }^{6}$ Each component of the type is known to the individual but is not verifiably observable by the insurance firm. Insurers believe the customer is of type $i \in T$ with probability $q_{i}$, where $q$ is an exogenously given prior.

Define the relevant action space as $A=\left\{(c, y) \in \mathbf{R}_{+}^{2}: c \leq b\right.$ and $\left.y \leq x\right\}$. For any contract $(c, y) \in A$ and customer of accident probability $\pi_{i} \in\left\{\pi_{L}, \pi_{H}\right\}$, a firm's expected profit is:

$$
V^{i}(c, y)=x-y-\left(1-\pi_{i}\right)(b-c)
$$

Thus when the agent is expected to be of either type with the prior probabilities $q$, expected profit is

$$
V^{q}(c, y)=q_{H} V^{H}(c, y)+q_{L} V^{L}(c, y) .
$$

The notion that an individual's degree of risk aversion is private knowledge is probably not controversial, inasmuch as it is certainly not directly observable. It might conceivably be argued that firms could elicit such information indirectly, to the extent needed, by requiring the individual to report her asset portfolio, for example. ${ }^{7}$ Even if the number of available signals is large, however, it is unlikely to equal or exceed the number of relevant characteristics in which informed agents can differ. Thus, in general, the case in which the dimensionality of private information exceeds that of signals warrants investigation.

\footnotetext{
${ }^{6}$ It might seem more natural to suppose that the two characteristics are distributed independently in the population, so that there are four types in place of the two considered here. Such an approach would not change the qualitative results of the analysis, since the single crossing property would hold between all pairs of types except the pair considered here. Extension to the case of more than two types over which the single crossing property cannot hold is more complicated, however, as the ensuing analysis should make clear.

${ }^{7}$ This possibility is considered explicitly by Laffont and Rochet (1988). Their analysis deals with a monopolistic insurer, however, rather than the competitive insurance industry considered here. See also Landsberger and Meilijson (1994) for an analysis of a monopoly insurance market with unobserved differences in risk aversion.
} 
This is particularly true when it is costly to verify reported actions. ${ }^{8}$

The relevance of risk aversion to the problem at hand is probably equally clear. In the standard explanation of screening in insurance markets, firms use deductibles on policies to sort between good and bad risks. In the present context, however, firms' statistical inference problems are more complicated: an individual who accepts a policy with a large deductible may be low risk (and therefore discounts the possibility of paying the deductible) or may be high risk, but relatively less averse to the possibility of paying the deductible. ${ }^{9}$

\subsection{Reactive equilibrium}

There has been considerable debate regarding the most appropriate notion of equilibrium for screening and signalling models. In introducing the screening concept, Rothschild and Stiglitz (1976) studied a two-stage game. In the first stage of the game, uninformed firms each offer a single policy, given prior beliefs about the distribution of types of potential customers; in the second stage, customers accept one of the policies on offer in the market. The authors then proceeded to seek subgame perfect equilibria in the game so defined. They demonstrated that a subgame perfect equilibrium is a maximal set of contracts that are profitable, given that insurance customers choose among contracts to maximize expected utility.

Subgame equilibria in pure strategies need not exist for the screening contract game. Non-existence arises when at the Pareto-dominant separating allocation there exist profitable deviation contracts that attract several types. Such deviations cannot themselves be equilibria, since given the single crossing condition there always exist further deviations attracting customers of higher profitability away from the original deviation and rendering it unprofitable. In order to address the problem of existence, a number of authors have proposed relaxations of the Nash equilibrium concept, which restrict the class of deviations to which an equilibrium must be immune. The most frequently employed of these is reactive equilibrium, proposed by Riley (1979). The logic of this concept is roughly that equilibria need not be immune to deviations which can themselves be undermined by further deviations.

To provide a formal definition of reactive equilibrium that is equivalent to Riley's, the following terminology is required. We denote the market offer set, the union of contracts offered by each firm, by $B \subseteq A$. For any market offer set $B$, define an individual's choice relation as:

$$
C^{i}(B) \equiv \inf _{c} \arg \max _{(c, y) \in B} U^{i}(c, y)
$$

\footnotetext{
${ }^{8}$ Crucial to these new qualitative results is the assumption that signals are scalars whereas individuals' types are ordered pairs. Earlier research on multidimensional signalling, including Kohlleppel (1983), Quinzii and Rochet (1985) and Engers (1987), has focussed on the case in which signals and types were of equal dimension greater than one and went on to derive sufficient conditions for equilibria to be separating and zero-profit. Closely related work by Milgrom and Roberts (1986) and Bagwell and Bernheim (1996) also studies separating equilibria when, for other reasons, the single crossing condition cannot hold.

${ }^{9}$ Note that risk aversion affects individuals' marginal rates of substitution, has no direct impact on firms' profits. Thus variable risk aversion effectively enters into a firm's statistical inference problem as noise that must be filtered to the extent possible.
} 
Let $(c, y) \equiv \alpha \in A$ be an arbitrary contract. The set of types choosing $\alpha$ from a set $B$ is denoted $T(\alpha, B)=\left\{i \in\{L, H\}: \alpha=C^{i}(B)\right\}$. We say $\alpha$ is profitable given $B$ and given beliefs $\hat{q}$ if

$$
\sum_{i \in T(\alpha, B)} \hat{q}_{i} V^{i}(\alpha) \geq 0
$$

That is, each contract at least breaks even on average, where expectation is over all types for which the contract is a most-preferred policy. A contract is strongly profitable given $B$ if it is profitable given any set that contains $B$. An allocation $B$ is said to be separating if, for all $\alpha \in B, V^{i}(\alpha) \geq 0$ for all types $i$ such that $\alpha=C^{i}(B)$. While this is perhaps not a very intuitive use of the term, such allocations are indeed "separating" inasmuch as no type is being cross-subsidized. Moreover, note that for an allocation that is separating and zero-profit two types accept the same contract $\alpha$ only if their gross profitabilities are equal at $\alpha$. This may correspond more readily to heuristic notions of separating allocations. ${ }^{10}$

Definition $1 A$ reactive equilibrium of the contract-offer game is a market offer set $B^{*} \subset$ A such that:

1. For any market offer $B$, informed individuals choose a contract to maximize utility on $B$.

2. $B^{*}$ is profitable.

3. For any contract $\alpha^{\prime} \in A \backslash B^{*}$, there exists a deviation $\alpha^{\prime \prime}$ that is strongly profitable given $B^{*} \cup\left\{\alpha^{\prime}\right\}$ such that $\alpha^{\prime}$ is not profitable given $B^{*} \cup\left\{\alpha^{\prime \prime}\right\}$.

The properties of reactive equilibria of the unidimensional screening game under single crossing are well known. It is straightforward to establish that under the single crossing condition there exists a unique reactive equilibrium of the contract-offer game, and that this equilibrium is the Pareto-dominant separating allocation.

Reactive equilibrium may be regarded by some as an unsatisfactory notion, since it is not readily seen to correspond to a particular Nash equilibrium refinement or a particular extensive-form representation of the contract-offer game. ${ }^{11}$ An alternative justification for reactive equilibrium can, however, be derived from the corresponding "take-it-or-leaveit" signalling game (Cho and Kreps, 1987) in which the informed insurance customer makes a contract offer to firms, which then accept or reject them conditional on posterior beliefs about the type of the customer making the offer. It can be demonstrated that the reactive equilibria of the screening game coincide with the perfect Bayesian equilibria of the signalling game which are consistent with the Cho-Kreps Intuitive Criterion. ${ }^{12}$

\footnotetext{
${ }^{10}$ In the terminology of Riley (1979), separating allocations are "incentive compatible" and profitable allocations are "weakly incentive compatible."

${ }^{11}$ Engers and Fernandez (1987) demonstrate that reactive equilibria are subgame perfect equilibria of an infinite-stage alternating-offer game among insurance companies. But, for reasons analogous to the Folk Theorem, there exists a multiplicity of other equilibria of the game they study.

${ }^{12}$ This equivalence is well known for single crossing preferences (see, e.g., Cho and Kreps, 1987), but can be shown to hold also in the absence of single crossing. In the interests of brevity, the proof is omitted here, but it is available from the author.
} 
Consequently, all of our results below for reactive equilibria can also be interpreted as applying to Intuitive equilibria of the signalling game. We will appeal to this equivalence below in interpreting some of the results.

\subsection{Equilibrium insurance contracts}

In the standard model where single crossing obtains, it is easily proven that the Paretodominant separating allocation represents the unique reactive equilibrium of the contractoffer game. To see this, note first that, given single crossing, a contract cannot pool two types in equilibrium: For any profitable pooling contract, there exists a deviation (with a larger deductible but smaller premium) which attracts only high-profit individuals and which therefore renders the original contract unprofitable. Moreover, this deviation cannot be undermined by further deviations, since it is separating and profitable, and therefore strongly profitable.

These arguments are dependent on the property that buyers' indifference curves are single crossing globally throughout the action space $A$. When both accident probabilities and degrees of risk aversion are unobserved by firms, this property no longer obtains globally on $A$. Rather, indifference curves are "double crossing" - viz. indifference curves of low risk, highly risk-averse individuals cross indifference curves of high risk, less risk-averse individuals twice (if they cross at all). It follows that it need not always be possible globally for firms to construct offer sets that separate these types of individuals. Specifically, in regions of the contract set $A$ in which high-profit individuals have a lower marginal rate of substitution $\sigma$, the two types cannot be pooled in equilibrium, since there will always exist deviations from pooling contracts that attract only the high-profit individuals. In contrast, in regions where low-profit agents have lower marginal rates of substitution, all profitable contracts that attract high-profit agents also attract low-profit agents, so that the possibility of pooling cannot be ruled out ex ante.

A preliminary step in describing equilibrium is therefore to characterize the set of contracts at which high-profit agents have lower marginal rates of substitution. ${ }^{13}$ To this end, let

$$
P=\left\{\alpha \in A: \sigma^{L}(\alpha)>\sigma^{H}(\alpha)\right\}
$$

denote the set of contracts at which low-profit agents have a lower marginal cost of signalling through the deductible than do high-profit agents. For reasons which will become clear, we will refer to $P$ as the "pooling set." Denote its complement as $S=A \backslash P$, which we will refer to as the "separating set." The boundary of $S$ on the interior of $A$ is therefore the locus of tangencies of the two types' indifference curves, defined by $\sigma^{L}(\alpha)=\sigma^{H}(\alpha)$, or

$$
\phi(c, y) \equiv \frac{\mu^{L}(c, y)}{\mu^{H}(c, y)}-\rho=0
$$

where

$$
\rho \equiv \frac{\pi_{H}\left(1-\pi_{L}\right)}{\pi_{L}\left(1-\pi_{H}\right)}>1 .
$$

\footnotetext{
${ }^{13}$ Here we follow the approach of Stiglitz and Weiss (1989).
} 


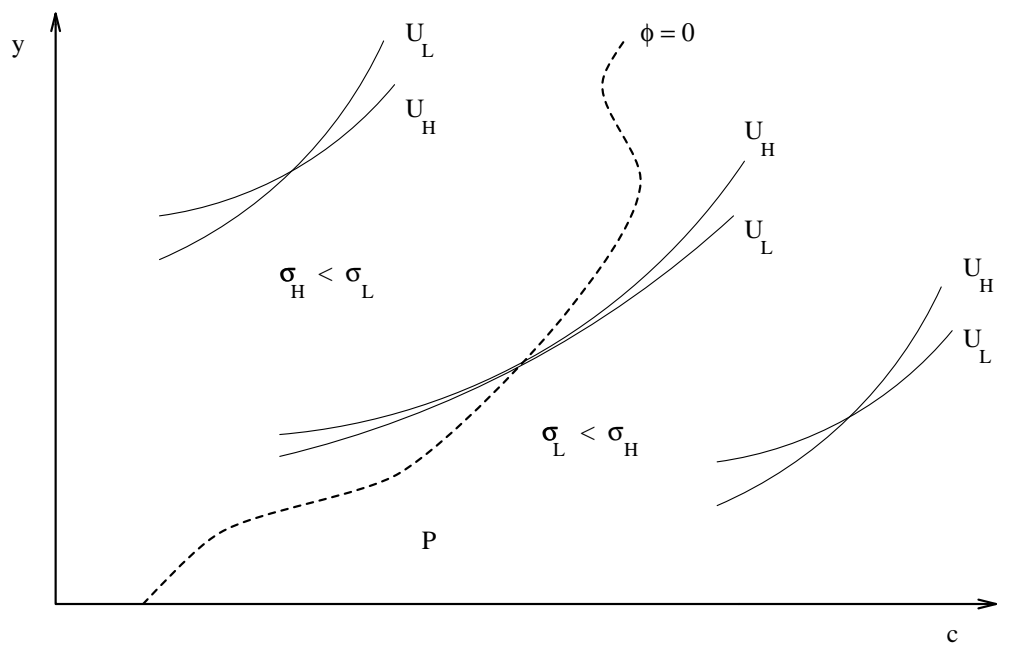

Figure 1: Regions of single crossing

From (3) and (7), the partial derivatives of $\phi$ are given by

$$
\begin{aligned}
& \phi_{c}(c, y)=\frac{\mu^{L}(c, y)}{\mu^{H}(c, y)}\left[a^{H}(y-c)-a^{L}(y-c)\right] \\
& \phi_{y}(c, y)=\frac{\mu^{L}(c, y)}{\mu^{H}(c, y)}\left[\left(a^{H}(y)-a^{L}(y)\right)-\left(a^{H}(y-c)-a^{L}(y-c)\right)\right] .
\end{aligned}
$$

Since risk aversion is increasing in $\theta$ by assumption, it follows that $\phi_{c}>0$ globally. Hence, by the Implicit Function Theorem, $\phi(c, y)=0$ defines $c$ as a continuously differentiable function of $y$, with

$$
\left.\frac{d c}{d y}\right|_{\bar{\phi}}=-\frac{\phi_{y}}{\phi_{c}}=1-\frac{a^{H}(y)-a^{L}(y)}{a^{H}(y-c)-a^{L}(y-c)} .
$$

Since risk aversion increases in $\theta, d c / d y<1$ along level sets of $\phi$.

Since this implicit function defines the boundary of $S$, it follows that $S$ is closed and arc-connected in $A$. Two other facts are relevant. First, $S$ contains the origin. Second, given concavity, the slopes of indifference curves are bounded above unity. Consequently, each indifference curve crosses the boundary of $S$ at most once. More formally, this condition implies the following.

Lemma 1 (i) For all $\alpha \in P$, for all $\alpha^{\prime} \geq \alpha, U^{H}\left(\alpha^{\prime}\right) \leq U^{H}(\alpha)$ implies $\alpha^{\prime} \in P$;

(ii) For all $\alpha \in S$, for all $\alpha^{\prime} \leq \alpha, U^{L}\left(\alpha^{\prime}\right) \geq U^{L}(\alpha)$ implies $\alpha^{\prime} \in S$.

Proof. See appendix.

It is this last observation that permits us to show that local conditions describing equilibria guarantee the existence and uniqueness of the equilibrium globally. Because of this identity between local and global conditions, $P$ is the set of contracts from which there exists no positive deviation which attracts high-profit but not low-profit individuals. 
Since it will be crucial in what follows, we state this property of the set $P$ formally as follows.

Lemma 2 For all $\alpha \in P$, for all $\alpha^{\prime}>\alpha, U^{H}\left(\alpha^{\prime}\right)>U^{H}(\alpha)$ implies $U^{L}\left(\alpha^{\prime}\right)>U^{L}(\alpha)$.

Proof. See appendix.

These observations are depicted in Figure 1. At contracts below and to the right of the broken line, the cost of signalling is less for individuals with high probability of an accident but low risk aversion. (This is the region we have denoted P.) The converse condition holds above and to the left of the broken line, which itself defines the locus of contracts at which $\phi(\alpha)=0$.

With this characterization of $P$, it follows that a contract which pools the two types cannot be undermined by deviations if and only if the contract is in $P$. Whether pooling occurs in equilibrium will therefore depend crucially on individuals' preferences for profitable contracts in $P$ and in $S$. This suggests two possible candidates for reactive equilibrium: (i) a separating equilibrium, in which incentive compatible, profitable separating allocations are offered, and (ii) a pooling equilibrium, in which incentive compatible, profitable pooling contracts are offered. We consider each of these sets in turn.

The natural candidate for a separating reactive equilibrium is the allocation that is Pareto dominant among incentive compatible, profitable separating allocations. This allocation,

$$
\begin{aligned}
& \left(\alpha_{0}, \alpha_{s}\right)=\arg \max _{\left(\alpha^{L}, \alpha^{H}\right)} U^{H}\left(\alpha^{H}\right) \\
& \text { s.t. } U^{L}\left(\alpha^{L}\right) \geq U^{L}\left(\alpha^{H}\right) \\
& \quad V^{i}\left(\alpha^{i}\right) \geq 0 \quad(i=L, H),
\end{aligned}
$$

is, in fact, the unique reactive equilibrium when preferences are single crossing in the traditional sense (Riley, 1979; Engers and Fernandez, 1987).

Different possible configurations for the allocation $\left(\alpha_{0}, \alpha_{s}\right)$ are depicted in Figure 2. The contract $\alpha_{0}$ is the most-preferred policy consistent with the non-negative profit condition for type $L$. Thus the policy offers full insurance with an actuarially fair premium for $L$; it is represented as point $D$ in Figure 2 . The contract $\alpha_{s}$, which is most preferred by type $H$, given the incentive constraint and the profit constraint, may correspond to points such as $A, B$, or $C$ in Figure 2-depending on the precise configuration of preferences and technologies. At point $A$, the optimum is a corner solution with both incentive and profit constraints binding. This is the only possibility in the usual analysis, where single crossing holds globally. If differences in risk aversion are sufficiently large (or differences in accident probability sufficiently small), however, the optimum occurs at an interior point such as $B$ or $C$ in Figure 2, where only the incentive constraint binds.

A simple argument establishes that, if the types are separated in reactive equilibrium, then the unique equilibrium allocation is $\left(\alpha_{0}, \alpha_{s}\right)$. In the terminology introduced in Section 2.2, the policy $\alpha_{0}$ is strongly profitable-viz. it earns non-negative expected profit regardless of beliefs about the type accepting the policy. Hence $\alpha_{0}$ can always be offered in reactive equilibrium. Conversely, no policy preferred to $\alpha_{0}$ can be offered to $L$ in a 


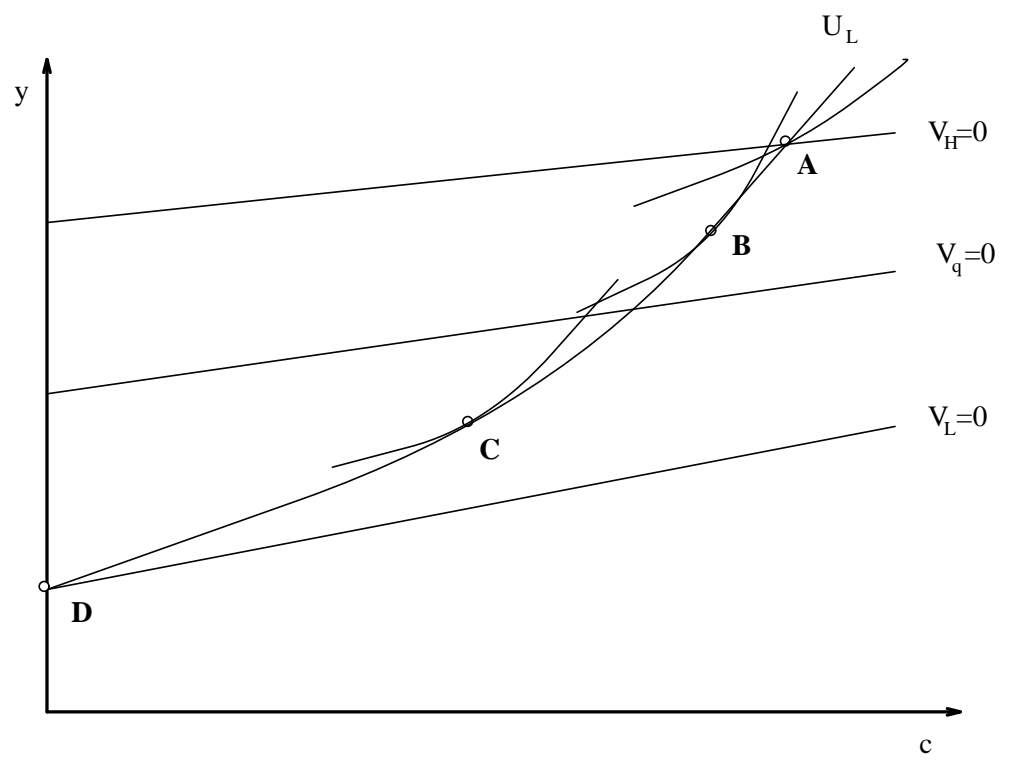

Figure 2: Preferences and equilibrium configurations

separating equilibrium, since such a policy would earn losses. Given that $L$ is offered $\alpha_{0}$ in equilibrium, moreover, $H$ must be offered $\alpha_{s}$. Any other policy would be undermined by a deviation to $\alpha_{s}$, or would violate incentive compatibility for $L$.

Thus, in seeking separating reactive equilibria in the model, one can confine attention to $\left(\alpha_{0}, \alpha_{s}\right)$. The following proposition establishes a sufficient condition for this allocation in fact to be a reactive equilibrium.

Proposition 1 If $V^{q}\left(\alpha_{s}\right) \leq 0$ then $\left(\alpha_{0}, \alpha_{s}\right)$ is a (separating) reactive equilibrium.

Proof. Consider any other profitable contract offer $\alpha^{\prime} \in A$. By construction, $\left(\alpha_{0}, \alpha_{s}\right)$ is Pareto dominant among separating allocations. Hence, if the deviation $\alpha^{\prime}$ is preferred by at least one type to $\left(\alpha_{0}, \alpha_{s}\right)$ and is profitable, then it must be preferred by both types. Since $\alpha^{\prime}$ is profitable, $V^{q}\left(\alpha^{\prime}\right) \geq 0$. Since $V^{q}\left(\alpha_{s}\right) \leq 0$ and $U^{H}\left(\alpha^{\prime}\right) \geq U^{H}\left(\alpha_{s}\right)$, moreover, it follows that $\alpha^{\prime}<\alpha_{s}$. (In words, if $H$ prefers a deviation with a larger premium, then the deviation must also have a smaller deductible.) Since $\alpha_{s} \in S$ by construction, $\alpha^{\prime} \in S$ by Lemma 1. Since $\alpha^{\prime}$ is in the separating set, then by the standard argument there exists a further profitable deviation $\alpha^{\prime \prime}$ with a slightly larger premium and smaller deductible that is strictly preferred to $\alpha^{\prime}$ by $H$, but not by $L$. This deviation $\alpha^{\prime \prime}$ is strongly profitable, since it attracts only $H$. Thus $\left(\alpha_{0}, \alpha_{s}\right)$ is a reactive equilibrium.

The proposition establishes that, whenever $V^{q}\left(\alpha_{s}\right)<0$, the constrained Pareto dominant separating allocation is a reactive equilibrium, in which $L$ accepts the offer of the policy $\alpha_{0}$ and $H$ accepts the offer of $\alpha_{s}$. When $V^{H}\left(\alpha_{s}\right)=0$, corresponding to point $A$ in Figure 2, we have the usual corner solution, in which insurers earn zero expected profit on the policies offered to each type in equilibrium. Note, however, that when $V^{H}\left(\alpha_{s}\right)>0$ (corresponding to point $B$ in Figure 2) insurers earn strictly positive expected profit on 
the policy accepted by $H$ in equilibrium. The profits are not offset by expected losses on the policy offered to $L$, since $V^{L}\left(\alpha_{0}\right)=0$ in any case. This establishes the following proposition.

Proposition 2 If $V^{q}\left(\alpha_{s}\right) \leq 0$ and $V^{H}\left(\alpha_{s}\right)>0$, then insurers earn strictly positive expected profit in reactive equilibrium.

This result is perhaps surprising, given the assumption that insurers compete as Bertrand-like price takers in the model. Thus it might be expected that competition among insurers would always drive excess profit to zero. (Indeed, some approaches to models of asymmetric information impose zero profit as a condition of equilibrium rather than derive it as a consequence.) But, under the conditions stated in the proposition, this need not occur. To see this, consider any potential deviation $\alpha^{\prime}$ from $\alpha_{s}$ with a lower premium and less excess profit. In this case, $\alpha_{s}$ is a point of tangency between indifference curves of the low-profit and high-profit types, corresponding to point $C$ in Figure 2. Hence if $H$ prefers $\alpha^{\prime}$ to $\alpha_{s}$ then $\mathrm{L}$ must also. Since $V^{q}\left(\alpha_{s}\right)<0$ by hypothesis, the deviation earns negative expected profit and will not be offered in equilibrium.

An alternative intuition can be developed by considering the corresponding "take-itor-leave-it" signalling formulation (Cho and Kreps, 1987) of the game, in which insurance customers propose contracts to insurers, which accept or reject offers based on posterior beliefs about the type of the customer making the offer. In a separating equilibrium, ${ }^{14}$ high-profit customers propose $\alpha_{s}$, their most-preferred policy consistent with incentive compatibility. In effect, high-profit agents choose a contract to distinguish themselves from low-profit agents in a least-cost fashion. In some cases (corresponding to point $A$ ) this is achieved using a deductible $c>0$ alone and an actuarially fair premium. If the difference in rates of absolute risk aversion of the two types is sufficiently large, however, signalling through the deductible is too costly at the margin. High-profit agents find it less expensive to signal type by offering an unfair policy (corresponding to point $B$ ).

In this interpretation, our results are similar to the model of advertising as a dissipative signal of product quality, proposed by Milgrom and Roberts (1986). In their work, producers of a high-quality product could signal type to consumers by setting a price that departed from the profit-maximizing level, or by engaging in advertising that was per se wholly uninformative. (Thus price corresponds to the costly signal through the deductible in the insurance model, whereas advertising corresponds to the purely dissipative signal through an actuarially unfair premium.) Milgrom and Roberts (1986) demonstrated that either price or advertising (or both) might be used to separate types in equilibrium. So here, both deductible and unfair premium may serve to screen in the insurance market.

The interpretation of the dissipative signal as advertising in Milgrom and Roberts (1986) and as an offer of excess profit in the insurance model is an arbitrary one, of course. The same effect could be achieved if type $H$ agents publicly burned money, for example. It is natural to think, however, that an offer of excess profit is the signal that is mostly easily verifiable for insurers.

Results thus far have established that when $V^{q}\left(\alpha_{s}\right)<0$ a separating equilibrium exists in the model. When $V^{q}\left(\alpha_{s}\right)>0$, in contrast, the possibility of a pooling equilibrium arises.

\footnotetext{
${ }^{14}$ Formally, we consider the unique perfect Bayesian equilibrium of the signalling game that is consistent with the Cho-Kreps Intuitive Criterion.
} 


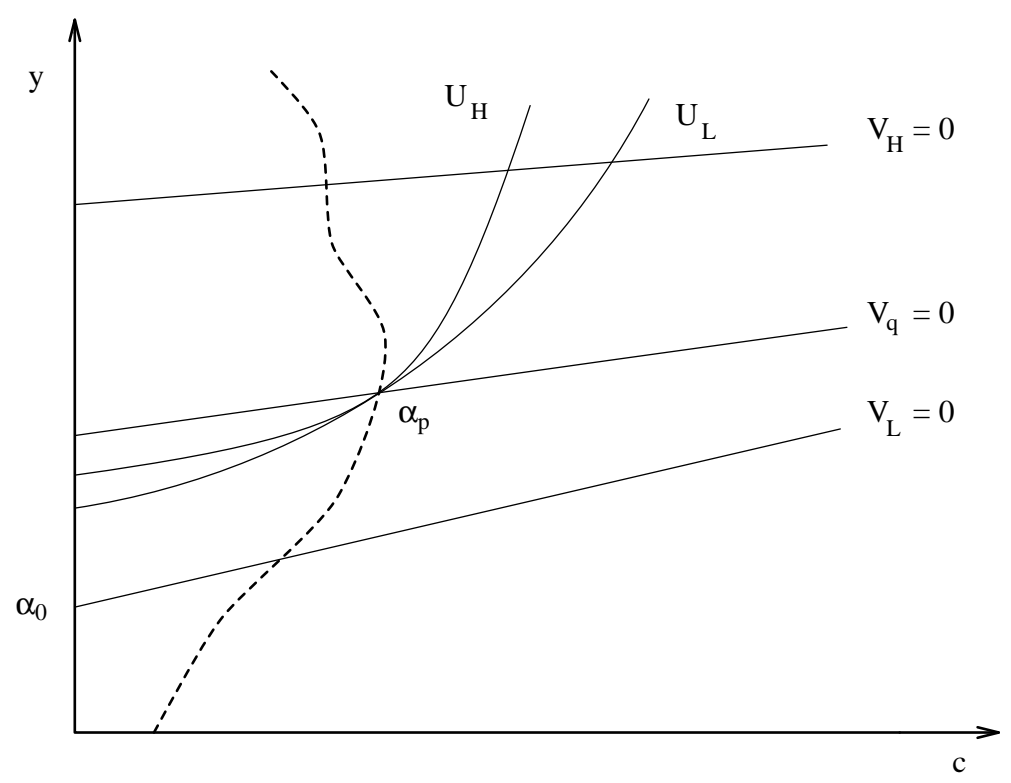

Figure 3: Equilibrium with pooling at $\alpha_{p}$.

Intuitively, when insurers earn positive expected profit on a pool of the two types at $\alpha_{s}$, then there must exist a profitable pooling contract that cannot be rendered unprofitable by a deviation attracting only the $H$ type. At the pooling equilibrium, any attempt to separate $H$ types with larger-deductible policies can be mimicked by $L$ types by virtue of their lesser aversion to risk.

To see this formally, define

$$
\alpha_{p}=\arg \max _{\alpha \in P} U^{L}(\alpha) \quad \text { s.t. } V^{q}(\alpha) \geq 0 .
$$

The policy $\alpha_{p}$ is not the Pareto efficient profitable pooling contract-characterized by full insurance for both types at a premium that is actuarially fair for the pool-since it is constrained to lie in the "pooling set" $P$ in which $L$ types have lower marginal cost of signalling through the deductible. By monotonicity of $U^{L}$, both constraints are binding at the optimum, so that $V^{q}\left(\alpha_{p}\right)=0$ and $\alpha_{p} \in$ bdy $P$, a point of tangency between indifference curves for the two types. This optimal policy is depicted in Figure 3.

Since $\alpha_{p}$ is a point of tangency between indifference curves for the two types, it is the allocation that is Pareto dominant among pooling allocations in the set $P$. Thus it is the natural candidate for a pooling equilibrium, when one exists. The following intuition confirms the intuition discussed above: when insurers could earn positive expected profit on a pool of the two types at the separating contract $\alpha_{s}$, then the separating equilibrium can be undermined by deviations, and $\left(\alpha_{p}, \alpha_{p}\right)$ is indeed a reactive equilibrium allocation.

Proposition 3 If $V^{q}\left(\alpha_{s}\right)>0$ then $\left(\alpha_{p}, \alpha_{p}\right)$ is a (pooling) reactive equilibrium.

Proof. By the foregoing argument, $\alpha_{p}$ is a point of tangency between indifference curves for types $L$ and $H$. Hence for all $\alpha^{\prime}, U^{H}\left(\alpha^{\prime}\right) \geq U^{H}\left(\alpha_{p}\right)$ implies $U^{L}\left(\alpha^{\prime}\right) \geq U^{L}\left(\alpha_{p}\right)$. Thus 
no deviation contract can attract $H$ without also attracting $L$. But if $\alpha^{\prime}$ is preferred by $L$ and $V^{q}\left(\alpha^{\prime}\right) \geq 0$ then $\alpha^{\prime} \in S$, since $\alpha_{p}$ is maximal in $P$. Hence there exists a strongly profitable deviation from $\alpha^{\prime}$, attracting only type $H$, which renders $\alpha^{\prime}$ unprofitable.

Suppose therefore there exists a profitable deviation $\alpha^{\prime}$ attracting only type $L$ from $\alpha_{p}$. Without loss of generality, we can set $\alpha^{\prime}=\alpha_{0}$, the most preferred contract that is profitable for $L$. By hypothesis $V^{q}\left(\alpha_{s}\right)>0$, and a fortiori $V^{H}\left(\alpha_{s}\right)>0$. Since the profit constraint for $H$ is slack in (9), $\alpha_{s}$ is also a point of tangency of indifference curves for the two types (see Figure 2). Hence $\alpha_{s} \in P$. By Lemma 1 , for all contracts $\hat{\alpha} \geq \alpha_{s}$ such that $U^{L}\left(\alpha_{s}\right) \geq U^{L}(\hat{\alpha}), \hat{\alpha} \in P$. It follows that $U^{L}\left(\alpha_{p}\right) \geq U^{L}\left(\alpha_{s}\right)=U^{L}\left(\alpha_{0}\right)$. This contradicts the supposition that $L$ preferred $\alpha_{0}$ to $\alpha_{p}$.

The preceding results have provided sufficient conditions for each of the three types of equilibrium - the separating equilibrium with zero and positive expected profit, and the pooling equilibrium - to exist. It is easily demonstrated that the equilibrium is also unique.

Proposition 4 A unique reactive equilibrium exists in the model. Equilibrium is separating (pooling) when $V^{q}\left(\alpha_{s}\right) \leq(>) 0$.

Proof. Existence of equilibrium follows from Propositions 1 and 3. To show uniqueness, first let $\left(\alpha_{0}, \alpha_{s}\right)$ be a reactive equilibrium allocation, and suppose that $V^{q}\left(\alpha_{s}\right)>0$. By the supposition, $\alpha_{s} \neq \alpha_{p}$. By Proposition $3, V^{q}\left(\alpha_{s}\right)>0$ implies $\left(\alpha_{p}, \alpha_{p}\right)$ is a reactive equilibrium. Hence $\alpha_{p}$ is a strongly profitable deviation from $\left(\alpha_{0}, \alpha_{s}\right)$ that attracts both types. This contradicts the definition of reactive equilibrium. Hence if $\left(\alpha_{0}, \alpha_{s}\right)$ is a reactive equilibrium then $V^{q}\left(\alpha_{s}\right) \leq 0$.

Conversely let $\left(\alpha_{p}, \alpha_{p}\right)$ be a reactive equilibrium, and suppose that $V^{q}\left(\alpha_{s}\right) \leq 0$. By Proposition $3, U^{L}\left(\alpha_{0}\right) \geq U^{L}\left(\alpha_{p}\right)$. Hence $\alpha_{0}$ is a strongly profitable deviation from $\left(\alpha_{p}, \alpha_{p}\right)$ (since by assumption $L$ chooses $\alpha_{0}$ in preference to $\alpha_{p}$ when $U^{L}\left(\alpha_{0}\right)=U^{L}\left(\alpha_{p}\right)$ ). This is again a contradiction; hence if $\left(\alpha_{p}, \alpha_{p}\right)$ is a reactive equilibrium then $V^{q}\left(\alpha_{s}\right)>0$.

\subsection{Efficiency of equilibrium and regulation}

Efficiency properties of equilibrium may also differ significantly from those obtained under single crossing. A typical property of reactive equilibrium under single crossing is that the equilibrium allocation is weakly constrained efficient, in the sense that it maximizes the utility of each type, subject to incentive compatibility constraints and the zero-profit conditions for each type. This property also holds for double crossing preferences, when the equilibrium is separating.

This notion of weak constrained efficiency must clearly be amended to deal with the case of pooling equilibrium, since in this case cross-subsidization occurs. A natural extension of this notion of weak efficiency is therefore that each contract in the allocation should earn non-negative profits. However, when the equilibrium involves pooling, the allocation may not be weakly constrained efficient in this modified sense. The inefficiency of the equilibrium results from the fact that incentive and feasibility constraints need not be binding at the optimal pooling contract $\alpha_{p}$. An example of this is depicted in Figure 3 , where the locus of tangencies of indifference curves $\phi(\alpha)=0$ intersects the zero-profit locus for pooling contracts $V^{q}(\alpha)=0$ in the interior of $A$. In this case, there exist profitable 
pooling contracts which Pareto dominate $\alpha_{p}$, but which are not attained by the reactive equilibrium allocation because such pooling contracts can be undermined by deviations.

When preferences are single crossing, in contrast, the separating reactive equilibrium has the property that the allocation is Pareto efficient given incentive constraints and given that cross-subsidization of types is not permitted. We define this notion of efficiency formally as follows. ${ }^{15}$

Definition 2 An allocation $\left(\hat{\alpha}^{L}, \hat{\alpha}^{H}\right)$ is weakly constrained efficient if, for $i=L, H, \hat{\alpha}^{i}$ solves

$$
\begin{aligned}
\max _{\left(\alpha^{L}, \alpha^{H}\right)} & U^{i}\left(\alpha^{i}\right) \\
\text { s.t. } & U^{i}\left(\alpha^{i}\right) \geq U^{i}\left(\alpha^{j}\right) \quad i, j=L, H \\
& V^{i}\left(\alpha^{i}\right) \geq 0 \quad i=L, H .
\end{aligned}
$$

An allocation is weakly constrained efficient if it is incentive compatible for both types and each contract in the allocation is weakly profitable. This is a fairly weak notion of efficiency, inasmuch as it does not permit cross-subsidization between types, even when this can relax incentive constraints and yield constrained Pareto improvements. An alternative notion of efficiency which does allow for cross-subsidization is the following.

Definition 3 An allocation $\left(\hat{\alpha}^{L}, \hat{\alpha}^{H}\right)$ is constrained efficient relative to population proportions q if, for $i=L, H, \hat{\alpha}^{i}$ solves

$$
\begin{array}{cl}
\max _{\left(\alpha^{L}, \alpha^{H}\right)} & U^{i}\left(\alpha^{i}\right) \\
\text { s.t. } & U^{i}\left(\alpha^{i}\right) \geq U^{i}\left(\alpha^{j}\right) \quad i, j=L, H \\
& q_{L} V^{L}\left(\alpha^{L}\right)+q_{H} V^{H}\left(\alpha^{H}\right) \geq 0
\end{array}
$$

Thus an allocation is said to be constrained efficient if it is incentive compatible and at least breaks even "on average," given the population proportions $q$.

Inspection of the definition of the equilibrium separating allocation (9) immediately indicates that $\left(\alpha_{0}, \alpha_{s}\right)$ is a weakly constrained efficient allocation. It is equally clear that the pooling equilibrium allocation cannot be weakly constrained efficient, since the nocross-subsidization constraints are violated by a pooling contract. The pooling allocation cannot be constrained efficient for any population proportions $q$, moreover, since both types receive a policy with less than full insurance, despite the fact that incentive constraints are not binding at $\alpha_{p}$. But Proposition 3 indicates that $\left(\alpha_{p}, \alpha_{p}\right)$ is a reactive equilibrium if and only if it Pareto dominates $\left(\alpha_{0}, \alpha_{s}\right)$. These observations establish the following characterization of the efficiency properties of equilibrium in the model.

Proposition 5 If the equilibrium allocation is separating, then it is weakly constrained efficient. If the equilibrium allocation is pooling, then it weakly Pareto dominates all weakly constrained efficient allocations. In either case, the equilibrium allocation is not constrained efficient.

\footnotetext{
${ }^{15}$ Our notions of efficiency correspond roughly to those proposed by Maskin and Tirole (1992).
} 
This suggests that reactive equilibrium need not satisfy any standard notion of constrained efficiency. Equilibrium pooling allocations weakly dominate all separating allocations, however. Thus pooling of types can occur in equilibrium only when pooling is welfare enhancing.

The notion that equilibrium is weakly constrained efficient even when it entails positive expected profit for insurers (as it does when the conditions of Proposition 2 hold) may be a surprising one, inasmuch as we study a partial-equilibrium model in which profits accruing to insurers are effectively "wasted" from the perspective of social welfare. But a positive-profit equilibrium occurs only when high-profit customers prefer accepting an unfair premium to accepting a large deductible; thus separation occurs in a least-cost fashion.

This observation suggests an alternative perspective on recent debates about insurers' profit and rate-of-return regulation in property-liability insurance markets. A frequently observed anomaly in liability insurance markets is the failure of insurers to exit from markets where premiums have been capped or rolled back through rate-of-return regulation by state governments, even when there is little evidence that insurers had earned monopoly rents prior to regulation. ${ }^{16}$ Harrington (1992) surveys the evidence for this phenomenon and suggests that insurers might find it optimal to remain in regulated markets, even when they are incurring losses, if (i) they expect regulatory constraints to be relaxed in the future and there are fixed costs to entering the market, or (ii) regulators use restrictions on other, profitable lines of insurance to prevent exit from the regulated liability line. But Proposition 2 suggests an alternative explanation. If insurers earn positive profit in competitive equilibrium because of the nature of incentive constraints, the optimal response to a regulated premium rollback is not to exit the market, but rather to offer policies with larger deductibles but smaller premiums, so that incentive constraints are again satisfied.

A further implication of the model is that, when excess profit accrues to insurers as a result of the nature of incentive constraints rather than non-competitive behaviour, rate-ofreturn regulation can have deleterious effects on social welfare. A regulatory intervention that serves to impose a zero-profit constraint induces an equilibrium allocation that is Pareto inferior to the unregulated equilibrium allocation. More formally, define a equilibrium with rate-of-return regulation to be a reactive equilibrium $B^{*}$ with the additional property that, for each contract $\alpha$ offered by insurers,

$$
\sum_{i \in T\left(\alpha, B^{*}\right)} q_{i} V^{i}(\alpha)=0
$$

That is, each contract offer is constrained to earn zero expected profit, conditional on the posterior distribution of types accepting the contract. This leads to the following result.

Proposition 6 Any equilibrium with rate-of-return regulation induces an equilibrium allocation which is weakly Pareto inferior to that obtaining in an unregulated market.

\footnotetext{
${ }^{16}$ Harrington (1992) writes, "the evidence on exits is somewhat puzzling. While significant exit has occurred in some states... it has not been truly rapid or widespread in any state ... . Another possibilitythat rates are suppressed, but only to levels that provide a fair rate of return - is also difficult to reconcile with long-run equilibrium in a competitively structured industry."
} 
Proof. Suppose that $V^{H}\left(\alpha_{s}\right)>0$ and $V^{q}\left(\alpha_{s}\right)<0$, so that by Proposition 2 insurers earn positive expected profit in an unregulated reactive equilibrium. (Otherwise, the constraint (11) is not binding.) In this case, the unique equilibrium with rate-of-return regulation consists of an offer of $\alpha_{0}$ for type $L$ and an offer of the contract consistent with the incentive and profit constraints that is most-preferred by type $H$. (This contract is depicted as point $A$ in Figure 2.) Note that any zero-profit deviation contract that is preferred by $H$ to this contract is also preferred by $L$. Hence, by the argument of Propositions 1 and 4, this allocation is the unique equilibrium.

\section{An explicit example}

The preceding analysis has demonstrated that equilibria can depart from that obtaining in the single crossing case-notably, pooling of types and positive profits for uninformed traders can occur-when unobservable types are two-dimensional and indifference curves cross twice. It might nevertheless be argued that the results are of little significance if such cases do not occur for economically reasonable parameterizations of profit and utility functions. In this section, a simple parameterization is adopted and computations are performed to demonstrate that the cases of interest are indeed reasonable ones. We will examine in particular the dependence of the equilibrium outcome on the degrees of risk aversion of the two types of insurance customer, performing simple comparative static calculations.

In the example, all individuals have constant absolute risk aversion preferences, so that

$$
u(z, \theta)=-e^{-\theta z} \quad \theta>0 .
$$

We again consider two types with rates of absolute risk aversion $\theta_{H}>\theta_{L}$ and accident probabilities $\pi_{H}>\pi_{L}$. The locus of tangencies of indifference curves for the types is then given by

$$
\phi(c, y)=e^{\left(\theta_{H}-\theta_{L}\right) c}-\rho=0,
$$

or, rearranging the expression,

$$
\bar{c}\left(\theta_{L}, \theta_{H}\right)=\frac{\log \rho}{\theta_{H}-\theta_{L}},
$$

where $\rho$ is given by (8), as before.

To derive explicit solutions for the equilibrium allocation in the model, note that, if the separating allocation $\left(\alpha_{0}, \alpha_{s}\right)$ is a reactive equilibrium then the low-profit type receives the zero-profit, full insurance contract $\alpha_{0}=\left(c_{0}, y_{0}\right)$, where $c_{0}=0$ and, from the zero-profit constraint for $L$,

$$
y_{0}=x-\left(1-\pi_{L}\right) b \text {. }
$$

The optimal separating contract for $H$ then solves the program (9). To characterize the solution, consider first the relaxed program, maximizing utility of type $H$ subject to the incentive constraint for type $L$,

$$
\begin{aligned}
& \max U^{H}\left(c_{s}, y_{s}\right) \\
& \text { s.t. } U^{L}\left(c_{0}, y_{0}\right) \geq U^{L}\left(c_{s}, y_{s}\right)
\end{aligned}
$$


A solution $\left(c_{s}, y_{s}\right)$ to (14) is a point of tangency of indifference curves for the two types, so that $c_{s}=\bar{c}\left(\theta_{L}, \theta_{H}\right)$ and, since the incentive constraint is binding,

$$
y_{s}=y_{0}+\frac{1}{\theta_{L}} \log \left[\pi_{L}+\left(1-\pi_{L}\right) e^{\theta_{L} \bar{c}}\right] .
$$

By Proposition 1 , if $V^{q}\left(c_{s}, y_{s}\right) \leq 0$, then a separating equilibrium exists. If $\left(c_{s}, y_{s}\right)$ satisfies the profit constraint for type $H$, so that

$$
V^{H}\left(c_{s}, y_{s}\right)=x-y_{s}-\left(1-\pi_{H}\right)\left(b-c_{s}\right) \geq 0,
$$

then $\left(c_{s}, y_{s}\right)$ solves the full program $(9)$ and is the equilibrium allocation, corresponding to point $B$ in Figure 2. If $V^{H}\left(c_{s}, y_{s}\right)<0$, conversely, then the profit constraint is binding at the equilibrium separating allocation-corresponding to point $A$ in Figure 2. The equilibrium contract offered to type $H$ is then the unique solution, say $\left(\tilde{c}\left(\theta_{L}\right), \tilde{y}\left(\theta_{L}\right)\right)$, to the incentive and profit constraints,

$$
\begin{aligned}
& \tilde{c}=b-\frac{x-\tilde{y}}{1-\pi_{H}} \\
& \tilde{y}=y_{0}+\frac{1}{\theta_{L}} \log \left[\pi_{L}+\left(1-\pi_{L}\right) e^{\theta_{L} \tilde{c}}\right] .
\end{aligned}
$$

To consider the final case, suppose that $V^{q}\left(c_{s}, y_{s}\right)>0$. Then, by Propositions 3 and 4 , the two types are pooled at the unique equilibrium allocation. Each type receives the policy $\left(c_{p}, y_{p}\right)$ with $c_{p}=\bar{c}\left(\theta_{L}, \theta_{H}\right)$, the deductible level at which the two indifference curves are tangent, and

$$
y_{p}\left(\theta_{L}, \theta_{H}\right)=x-\left(1-\pi_{q}\right)\left(b-\bar{c}\left(\theta_{L}, \theta_{H}\right)\right),
$$

where $1-\pi_{q}$ is the probability an accident occurs given that the customer is believed to be type $L$ with probability $q_{L}$ and type $H$ with probability $q_{H}$.

Collecting these observations, the equilibrium policy offered to type $H$ is given by

$$
c^{*}\left(\theta_{L}, \theta_{H}\right)= \begin{cases}\tilde{c}\left(\theta_{L}\right) & \text { if } V^{H}(\bar{c}, \bar{y})<0 \\ \bar{c}\left(\theta_{L}, \theta_{H}\right) & \text { otherwise }\end{cases}
$$

and

$$
y^{*}\left(\theta_{L}, \theta_{H}\right)= \begin{cases}x-\left(1-\pi_{H}\right) \tilde{c}\left(\theta_{L}\right) & \text { if } V^{H}(\bar{c}, \bar{y})<0 \\ \bar{y}\left(\theta_{L}, \theta_{H}\right) & \text { if } V^{H}(\bar{c}, \bar{y}) \geq 0 \text { and } V^{q}(\bar{c}, \bar{y}) \leq 0 \\ x-\left(1-\pi_{q}\right) \bar{c}\left(\theta_{L}, \theta_{H}\right) & \text { if } V^{q}(\bar{c}, \bar{y})>0\end{cases}
$$

To solve numerically for the functions, an illustrative set of parameter values has been chosen. Suppose that the insurance customer faces a potential income loss of $\$ 5,000$. Lowprofit types experience the loss with probability one-half $\left(\pi_{L}=0.5\right)$ whereas high-profit agents experience the loss with probability $0.3\left(\pi_{H}=0.7\right)$. Insurers' prior belief is that a customer is type $L$ with probability 0.8 and type $H$ otherwise, so that the prior probability of a loss is $\pi_{q}=0.54$. 


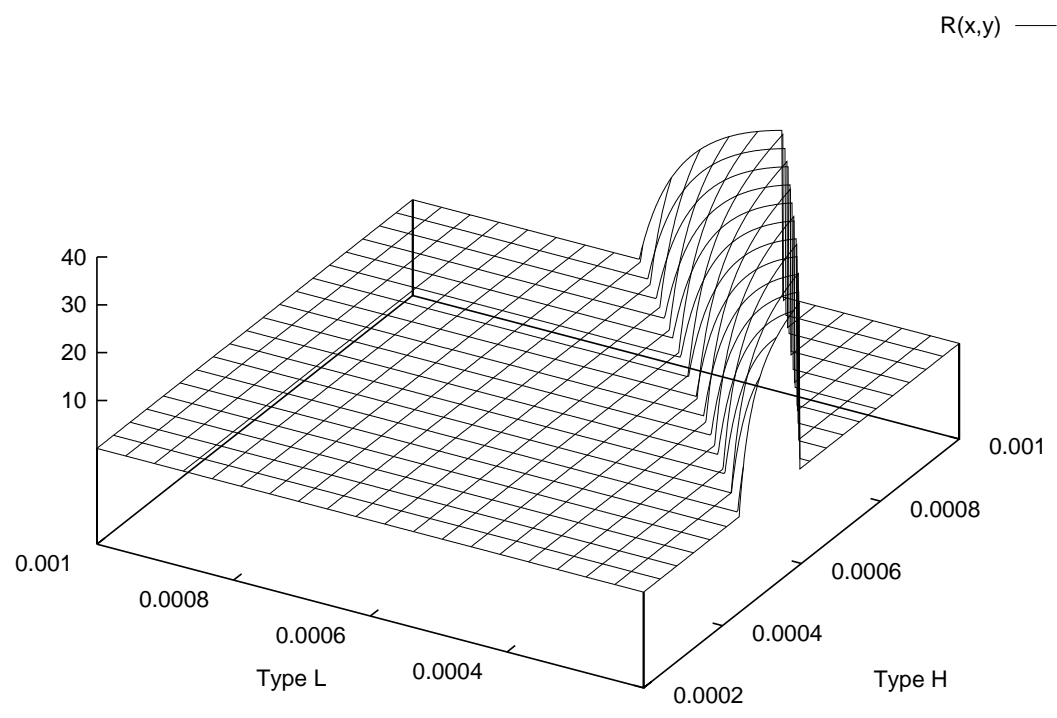

Figure 4: Excess profit as a function of $\left(\theta_{L}, \theta_{H}\right)$.

The key parameters of interest are the absolute rates of risk aversion of the two types of customer, $\left(\theta_{L}, \theta_{H}\right)$. To examine how the equilibrium changes with these parameters, we let $\theta_{L}$ range between $2 \times 10^{-4}$ and $1 \times 10^{-3}$, while $\theta_{H}$ ranges between $\theta_{L}$ and $1 \times 10^{-3}$. To understand better the economic implications of these parameter ranges, note that, when $\theta_{L}=2 \times 10^{-4}, L$ 's risk premium for the $\$ 5,000$ gamble is $\$ 600$, whereas when $\theta_{L}=1 \times 10^{-3}$, $L$ 's risk premium is $\$ 1,814$.

The comparative statics of equilibrium with respect to $\left(\theta_{L}, \theta_{H}\right)$ are straightforward. Given $\theta_{L}$, as $\theta_{H}$ increases, the equilibrium allocation alters smoothly from the zero-profit separating equilibrium (point $A$ in Figure 2) to the positive-profit separating equilibrium (point $B$ in Figure 2) to the pooling equilibrium (point $C$ in Figure 2). Hence the equilibrium deductible for $H$ is monotone non-increasing in $\theta_{H}$. Equilibrium excess profit initially increases in $\theta_{H}$ over the parameter range inducing the positive-profit separating equilibrium, and then decreases discontinuously to zero for the parameter range which induces the pooling equilibrium.

These results are as expected: For small differences in risk attitudes of the agents, high-profit agents are able to signal in the usual fashion by accepting a policy with a larger deductible; hence the usual zero-profit separating equilibrium obtains. For greater differences in risk attitudes, high-profit agents prefer to signal at the margin by accepting increases in the premium that are actuarially unfair, and the positive-profit equilibrium obtains. Finally, given large differences in risk attitudes of the two types, the high-profit type is unwilling to signal at all in order to be distinguished from the low-profit type. Thus the two types are pooled in equilibrium.

The numerical calculations indicate that all three types of equilibrium allocation can 
occur for reasonable parameter values, and that equilibrium excess profit can be substantial. Excess profit as a percentage of the premium is graphed as a function of $\left(\theta_{L}, \theta_{H}\right)$ in Figure 4. To interpret the Figure, note that, when $L$ 's risk premium is $\$ 600$, the positiveprofit equilibrium occurs for values of $\theta_{H}$ yielding a risk premium for type $H$ of $\$ 1,297$. The pooling equilibrium occurs for risk premiums greater than $\$ 1,663$. Similarly, when $L$ 's risk premium is increased to $\$ 1,814$, the positive-profit equilibrium exists for values of $\theta_{H}$ yielding risk premia between $\$ 2,629$ and $\$ 2,808$, and the equilibrium is pooling for all higher values of $\theta_{H}$. Excess profit rises to equal approximately 35 per cent of premium revenue for the largest values of $\theta_{H}$ consistent with the positive-profit equilibrium.

These calculations are intended only to be illustrative, of course, and have little predictive content about the nature of equilibria to be observed in actual insurance markets. It seems reasonable to infer, however, that even relatively small differences in risk attitudes among insurance customers can lead to equilibrium outcomes that overturn the usual predictions of models of screening or signalling in insurance markets.

\section{Conclusion}

The assumption of unidimensionality in asymmetric information models has few forceful apologists. In most cases of economic interest, it is generally accepted that several relevant characteristics of market participants are private information, and indeed that the dimensionality of characteristics may well exceed the dimensionality of signalling actions available to participants. Unidimensionality is therefore often regarded as a necessary simplification of the model that generates results that are salient to more general environments.

The analysis in this paper suggests that such views may be ill-founded. In a canonical application of the theory -insurance markets - the addition of a second dimension to informed agents' characteristics can change the nature of reactive equilibrium in significant ways. In contrast to the zero-profit separating allocation that is the reactive equilibrium of the usual unidimensional case, the unique equilibrium for the two-dimensional case may involve pooling of types, and positive profits earned by uninformed firms. The efficiency properties of equilibrium may also differ from those obtaining under single crossing.

\section{A Appendix}

\section{Proof of Lemma 1}

(i) Since $U^{H}$ is continuously differentiable, we can choose a monotone, differentiable path $\alpha(p)$ from $\alpha$ to $\alpha^{\prime}$ such that

$$
\frac{d}{d p} U^{H}(\alpha(p))=\left[c^{\prime}(p)-\sigma^{H}(\alpha(p)) y^{\prime}(p)\right] U_{c}^{H}(\alpha(p))<0 .
$$

Hence $c^{\prime}-\sigma^{H} y^{\prime}>0$, since $U_{c}^{H}<0$, and

$$
\frac{d \phi}{d p}=\left(c^{\prime}-\left.\frac{d c}{d y}\right|_{\bar{\phi}} y^{\prime}\right) \phi_{c}>0
$$


since

$$
\sigma^{H}>1>\left.\frac{d c}{d y}\right|_{\bar{\phi}}
$$

and $\phi_{c}>0$. It follows that $\phi\left(\alpha^{\prime}\right)>\phi(\alpha)$, so that $\alpha \in P$ implies $\alpha^{\prime} \in P$.

The proof of part (ii) is analogous.

\section{Proof of Lemma 2}

Choose $\alpha^{0} \in P$ and $\alpha^{1}>\alpha^{0}$ such that $U^{H}\left(\alpha^{1}\right)>U^{H}\left(\alpha^{0}\right)$. Suppose the Lemma is false, so that $U^{L}\left(\alpha^{1}\right) \leq U^{L}\left(\alpha^{0}\right)$. Hence, by virtue of Lemma $1, \alpha^{1} \in P$. Since $\alpha^{1}>\alpha^{0}$, there exists a monotone, differentiable path $\alpha(p)=(y(p), c(p)), p \in[0,1]$, such that $\alpha(p) \in P$ and

$$
\frac{d}{d p} U^{H}(\alpha(p))=\left[c^{\prime}(p)-\sigma^{H}(\alpha(p)) y^{\prime}(p)\right] U_{c}^{H}(\alpha(p))>0
$$

for all $p$. Since $\alpha(p) \in P$,

$$
c^{\prime}(p)-\sigma^{L}(\alpha(p)) y^{\prime}(p) \geq c^{\prime}(p)-\sigma^{H}(\alpha(p)) y^{\prime}(p)>0,
$$

implying

$$
\begin{aligned}
U^{L}\left(\alpha^{1}\right)-U^{L}\left(\alpha^{0}\right) & =\int_{0}^{1} \frac{d}{d p} U^{L}(\alpha(p)) d p \\
& =\int_{0}^{1}\left[c^{\prime}(p)-\sigma^{L}(\alpha(p)) y^{\prime}(p)\right] U_{c}^{L}(\alpha(p)) d p>0,
\end{aligned}
$$

which contradicts the supposition. 


\section{References}

Bagwell, L. S. and B. D. Bernheim (1996): "Veblen effects in a theory of conspicuous consumption," American Economic Review, Forthcoming.

Cho, I. K. And D. KRePs (1987): “Signaling games and stable equilibria," Quarterly Journal of Economics, 102, 179-221.

Engers, M. (1987): "Signalling with many signals," Econometrica, 55, 663-674.

Engers, M. and L. Fernandez (1987): "Market equilibrium with hidden knowledge and self-selection," Econometrica, 55, 425-439.

Harrington, S. E. (1992): "Rate suppression," Journal of Risk and Insurance, 59, $185-201$.

Kohlleppel, L. (1983): "Multidimensional market signalling," Discussion Paper 125, Universität Bonn.

LAFFont, J.-J. AND J.-C. Rochet (1988): "Stock market portfolios and the segmentation of the insurance market," Scandinavian Journal of Economics, 90, 435-446.

Landsberger, M. And I. Meilijson (1994): "Monopoly Insurance under Adverse Selection When Agents Differ in Risk Aversion," Journal of Economic Theory, 63, 522-531.

Maskin, E. And J. TiRole (1992): "The principal-agent relationship with an informed principal, II: Common values," Econometrica, 60, 1-42.

Milgrom, P. And J. Roberts (1986): "Price and advertising as signals of product quality," Journal of Political Economy, 94, 796-821.

Miyazaki, H. (1977): "The rat race and internal labor markets," Bell Journal of Economics, 8, 394-418.

Myerson, R. B. (1983): "Mechanism design by an informed principal," Econometrica, $51,1767-1798$.

QuinziI, M. And J.-C. Rochet (1985): "Multidimensional screening," Journal of Mathematical Economics, 14, 261-284.

Riley, J. (1979): “Informational equilibrium,” Econometrica, 47, 331-360.

Rothschild, M. and J. E. Stiglitz (1976): "Equilibrium in competitive insurance markets: An essay on the economics of imperfect information," Quarterly Journal of Economics, 90, 629-649.

Stiglitz, J. E. And A. Weiss (1989): "Credit rationing and its implications for macroeconomics," Mimeograph, Stanford University.

Wilson, C. (1977): “A model of insurance markets with incomplete information," Journal of Economic Theory, 16, 167-207. 